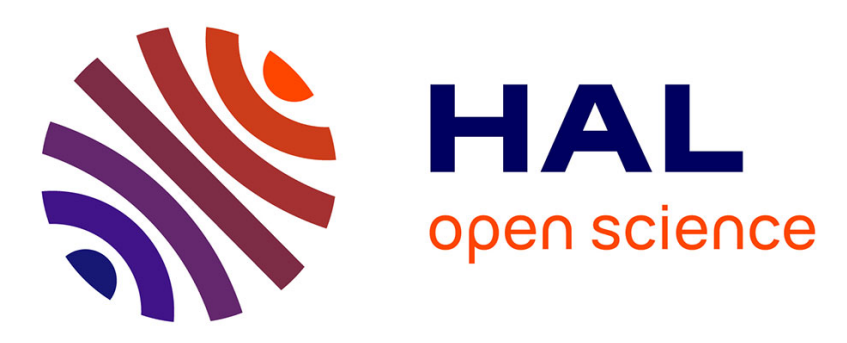

\title{
TAR channel access mechanism for VANET safety-critical situations
}

Ines Khoufi, Bachar Wehbi, Anis Laouiti

\section{To cite this version:}

Ines Khoufi, Bachar Wehbi, Anis Laouiti. TAR channel access mechanism for VANET safety-critical situations. CIAS 2012: 4th International Conference on Intelligent and Advanced Systems, Jun 2012, Kuala Lumpur, Malaysia. pp.226 - 231, 10.1109/ICIAS.2012.6306192 . hal-00864215

\section{HAL Id: hal-00864215 https://hal.science/hal-00864215}

Submitted on 12 Dec 2013

HAL is a multi-disciplinary open access archive for the deposit and dissemination of scientific research documents, whether they are published or not. The documents may come from teaching and research institutions in France or abroad, or from public or private research centers.
L'archive ouverte pluridisciplinaire HAL, est destinée au dépôt et à la diffusion de documents scientifiques de niveau recherche, publiés ou non, émanant des établissements d'enseignement et de recherche français ou étrangers, des laboratoires publics ou privés. 


\title{
TAR Channel Access Mechanism For VANET Safety-Critical Situations
}

\author{
Ines Khouf*, Bachar Wehbi ${ }^{\dagger}$, Anis Laouiti ${ }^{\ddagger}$ \\ *ines.khoufi@it-sudparis.eu, †bachar.wehbi@montimage.com, ${ }^{\ddagger}$ anis.laouiti@it-sudparis.eu \\ $* \ddagger$ TELECOM SudParis/9 rue Charles Fourier 91011, Evry Cedex, France \\ ${ }^{\dagger}$ Montimage/39 rue Bobillot 75013, Paris, France
}

\begin{abstract}
Vehicular Ad-hoc Network (VANET) is among the most relevant forms of mobile ad-hoc networks. VANET helps improving traffic safety and efficiency. By exchanging information between each others, vehicles can warn drivers or even prepare for dangerous situation. These warnings can be about critical situations like vehicles merging in a highway. Detecting and warning about such situations require a reliable communication between vehicles increasing thus the need for an efficient medium access control (MAC) protocol. In this paper, we propose to apply Transmit And Reserve (TAR), an adhoc medium access protocol, to vehicular communications. We integrated TAR into NS-3 simulator and evaluated its performance compared to IEEE 802.11 DCF in a vehicular network context. The evaluation results show that TAR is an efficient medium access protocol for VANET critical situations as it increases the throughput reduces the medium access delays and provides close to optimal short term fairness.
\end{abstract}

\section{INTRODUCTION}

Vehicular communication is a key technology in intelligent transportation systems. For many years now, the academic and industrial research communities have been investigating these communications in order to improve efficiency and safety of future transportation. VANET is one special kind of mobile ad-hoc networks to provide Vehicle to Vehicle (V2V) or Vehicle to Infrastructure (V2I) communications. VANET is a dynamic high density network characterized by quasi permanent mobility, high speed and very short connection times between vehicles. Safety vehicular communications require very high data transfer, low collision rates, low latency, fair share of throughput and fair medium access. For a successful deployment of VANET, an efficient medium access control (MAC) protocol for timely and reliable messages exchange is needed. Wireless Access in Vehicular Environments (WAVE) [1] is a key enabling technology for VANET applications and services. WAVE architecture use IEEE 802.11 Carrier Sense Multiple
Access/Collision Avoidance (CSMA/CA) mechanism for medium access control based on IEEE 802.11 DCF method. Nevertheless, IEEE 802.11 access scheme is not efficient enough for safety communication in vehicular network [2]. In this work, we consider the application of Transmit And Reserve (TAR) [3] to VANET safety critical situations. TAR is a wireless networking medium access mechanism that optimizes the throughput, reduces the number of collisions and provides high short term fairness. These TAR characteristics make it a potential candidate for vehicular communications. In this perspective, we studied its performance and compared it against IEEE 802.11 medium access protocol in a VANET safety critical situation.

This paper is organized as follows: In section II we present our motivations for this work and we list some related work. In section III, we describe TAR mechanism. In section IV, we start by fixing the parameters of our simulation scenario, and then we provide the performance evaluation of TAR compared to IEEE 802.11 DCF. Finally we conclude in section V.

\section{Motivation}

One of the main objectives of a VANET is to provide communication means in order to increase safety on the roads and minimizes damages in case of dangerous situations. When we are driving a car, two types of dangerous situations can occur: emergency situation or critical situation.

An emergency situation is defined as a situation with an immediate danger of collision, like the existence of an obstacle on the road. Notice that the obstacle in itself could be the result of a previous collision of two or more cars. The drivers of the vehicles coming behind may not avoid additional accidents if they are not warned in time about this danger.

The critical scenario could be seen as an apparently safe situation where a regular traffic is flowing without 


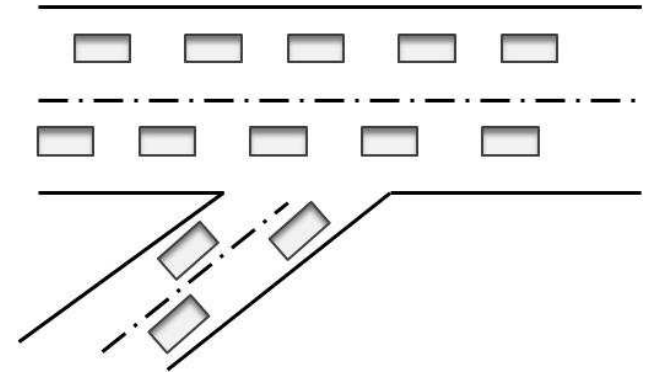

Fig. 1. Example of vehicular critical situation

any immediate danger (i.e. no obstacle ahead or any other event that has originated an accident). However, a non respect of a priority due to a lack of visibility of one car driver could be dramatic and results in an accident. A vehicle merge in a highway is an example of such situations (see Fig. 1), a car driver have to pay attention and keep an eye to the cars in front and behind to maintain a safe distance, as well as to the cars going in the main lane while identifying the gap between cars and the right moment where the merge is safe. All these tasks must be executed within seconds by each driver in order to maintain a fluent traffic. A priority violation, a bad speed increase or decrease may provoke a collision. In these situations we should insure a high level of coordination between cars in order to manage the traffic flow and avoid incidents. The drivers involved in car merge situations, should be warned on time and coordinate between each others to agree on the speed of each vehicle.

In a typical emergency scenario like a car collision in a highway, the main goal of $\mathrm{V} 2 \mathrm{~V}$ communications is to diffuse this information to warn the car drivers behind travelling at high speed about the immediate danger. In this scenario the damaged vehicles will start generating emergency messages which must be broadcasted and relayed in a multihop manner as quickly as possible. It is a type of multicast communication (one source and many receivers) that is used to flood this emergency information within the vehicular network. Many flooding techniques are proposed in the literature where the most important factors to optimize are the delivery delay as well as the bandwidth cost. In [5], authors show that a technique as simple and basic as simple flooding outperforms other more complicated schemes. The major drawback remains the excessive bandwidth consumption, but, in these scenarios one could accept this high cost if the objective is to save human lives.

Critical safety scenarios are different essentially because there are many stakeholders, and each of them must be granted the right to send and receive safety messages in a short time.

This work considers vehicular critical situations which can lead to vehicular emergency situations. In these situations, the communication protocol is expected to provide high channel access guarantees with low delays. In fact, in critical situations we need better coordination between communicating vehicles in order to collect transmitted data from all of them and allow the drivers to take the best decisions as well as insuring other applications data communication without interruption. The key solution to meet these requirements is an adapted channel access approach that provides low access delays and high fairness among participating nodes.

WAVE relies on the standard IEEE $802.11 \mathrm{p}$ to organize the channel access between the participating members. As the channel access in this standard is based on the CSMA/CA principle and uses the Binary Exponential Backoff (BEB) to handle the collisions and reduce their effect, it suffers from several shortcomings. BEB has a number of drawbacks. First, it does not eliminate the collisions, but rather tends to reduce their effect. Then, fairness is also one of the important issues in $\mathrm{BEB}$, as it tends to favor the last contention winner (or new contending nodes) over other contending nodes, when allocating channel resources. The new contending nodes (as well as the last contention winner) select their Backoff value from the minimum contention window (CW) value. The other contending nodes must decrement their values, slot by slot before accessing the channel, and if their transmission fails they have to double the contention window before choosing a new random Backoff value. This process is repeated for a predefined maximum number of times before rejecting the packet. Collisions and backoff delays can be harmful for VANET communications in safety critical scenarios. Loss or late delivery of safety messages as well as channel access unfairness have strong impact on the safety applications efficiency which will be unable to inform the drivers of dangerous situations and let them react on time. Some studies try to solve the IEEE 802.11 channel access problems by proposing algorithms to adjust the Backoff contention window [4] [6] or by enhancing CSMA scheme's with new mechanism [6] to improve throughput and packet reception rate. Other works have proposed a deterministic access for IEEE 802.11p [7] in order to provide a bounded delays for emergency messages. These solutions provide an enhancement over IEEE 802.11 channel access but they don't always perform 


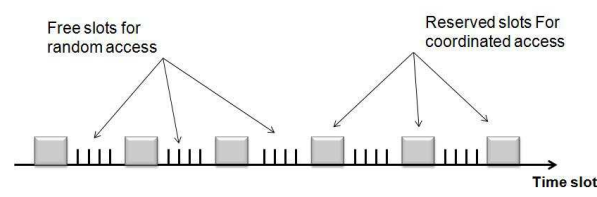

Fig. 2. TAR Channel Access

well in a dense network and most of the time the short term fairness of the channel access, a required property in vehicular communication, is simply not studied.

In a previous work, Transmit And Reserve (TAR) protocol [3] has been proposed to organize the channel access and improve the channel utilization and the short term fairness. This protocol tends to cope with the above cited problems of the BEB algorithm.

In this work we are evaluating TAR in a scenario of vehicular critical situation. Our objective is to study the performance of TAR in a V2V environment. We have implemented TAR using NS-3 simulator. Then we have studied its performance in vehicular critical situation scenario by comparing it to IEEE 802.11 DCF mode.

\section{TAR: TRANSMIT AND RESERVE}

Transmit And Reserve is a per-packet coordinated channel access scheme for IEEE 802.11 wireless networks. The objective of TAR is to define an access method that optimizes the throughput, reduces the number of collisions and provides high short term fairness. The idea of TAR is to create a cycle of active nodes accessing sequentially to the channel. The IEEE 802.11 MAC is based on the CSMA/CA random access. This method allows a node to select random Backoff of slots before attempting to transmit. But this can produce collisions when two or more nodes select the same Backoff value at a given moment. The key idea of TAR is to support the network nodes with the means to avoid selecting Backoff values that are already selected by other nodes. Consequently, the major reason for collisions will be eliminated. For that, TAR defines a mechanism that consists of:

- Selecting in advance a free Backoff value for the next packet waiting for transmission,

- Advertising (reserving) the selected Backoff value within the current packet to transmit,

- Maintaining a Backoff Reservation counter in order to track the advertised (reserved) Backoff values in the network.
Fig. 2 illustrates TAR's channel access organization. Using TAR mechanism, the network will be organized into a cycle in which each active node accesses once to the channel. The cycle resulting from the advertisement of selected backoff values and the synchronization of the maintained Backoff Reservation counters to the most upto-date advertisement. Each node in TAR cycle maintains separately a common counter; by synchronizing their Backoff Reservation counters to the same received advertisement a distributed coordination of channel access is provided. When a node is granted to transmit a packet, it updates its Backoff Reservation counter value by adding to it a predefined step parameter value with step $>2$. Then, it sets the Backoff value for the next packet waiting for transmission to the updated Backoff Reservation counter and advertises this selected Backoff in the current packet to transmit.

When neighbor nodes receive this advertisement, they update their Backoff Reservation counters to fall back again on a common value within the local neighborhood network. If an idle slot is sensed on the channel, the node decrements by one the value of its Backoff and Backoff Reservation counters. As the step parameter value $>2$, some free slots will occur between two consecutive TAR reservations. This allows new nodes to join the TAR cycle by randomly choosing a Backoff value from the gaps of free slots between the reserved selected Backoff values. TAR access scheme can be summarized into two phases. The first is random access phase where a new node joins the coordinated transmission cycle and the second is coordinated access phase where a node participates in the TAR access cycle as long as it has more packets waiting for transmission.

In fig. 3 we give an example of TAR mechanism and show how two new nodes join the TAR cycle. In this example, a TAR cycle exists already and it is composed by node 1 and node 2 . These nodes are exchanging packets and advertising a Backoff Reservation (BOR) equal to 10 slots for their next transmissions respectively. When node 3 has packets to send, it joins the cycle by selecting a random Backoff from the available free slots that are between two sccessives transmissions. When node 3 is granted channel access to transmit its packets it will advertise a value of BOR equal to BOR+step (step is equal to 5 slots in our work). Node 1 and node 2 receive this value which is higher than the maintained Backoff reservation, so they update their BOR (the BOR value will be equal to 15 slots for a network of three nodes). This value is maintained by all active nodes in TAR cycle. When node 4 has packet to send, the same scenario 


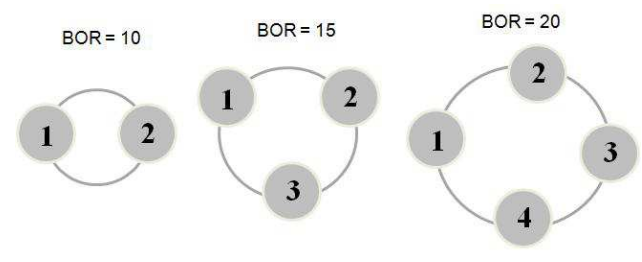

BOR : Counter of the Backoff reservation maintained by all nodes of the cycle

Fig. 3. Example of TAR Mechanism

will be repeated. First it joins the cycle by selecting a random Backoff from free slots. Second it executes the coordination phase, and advertises a new BOR equal to $\mathrm{BOR}+$ step. Finally these four nodes will advertise the value of 20 slots for their next transmissions.

\section{TAR EVALUATION}

In order to evaluate TAR mechanism in a VANET network, we integrated it in the MAC Layer of NS-3 simulator [9]. Then, we compared TAR to IEEE 802.11 DCF mode. The number of nodes in the simulated scenario varied from 5 to 100 nodes with a fixed throughput of $11 \mathrm{Mbps}$. For every density, 20 different simulations have been carried out and the results averaged. We generated 1 packet each 0.001 seconds, to have the buffer at link layer never empty. The packet size at MAC layer was set to 1500 bytes. In this scenario all nodes are neighbors and are in the same range, so we eliminate the risk of the propagation loss. The RTS/CTS handshake of the MAC layer in this set of simulations was disabled. The simulation time was set to 100 seconds, which is a sufficient duration to observe the mechanism of both TAR and IEEE 802.11 DCF.

In this section we evaluate the performance of TAR based on the following criteria: the network throughput, the collision rate, the short term fairness based on Jain's fairness index [8] and the inter-transmission delay. In addition we compare the results of TAR mechanism to those of DCF mode to show the behavior of TAR in a Vehicular communication in critical situations.

In this work, TAR shows some differences in the simulation results in comparison to the previous work results, because we are evaluating it using simulation parameters which are more appropriate to vehicular communications. However, these differences do not affect neither TAR's behavior nor its performance.

\section{A. Throughput}

Fig. 4 compares the throughput of TAR and IEEE 802.11. We can see that in a low density networks, the

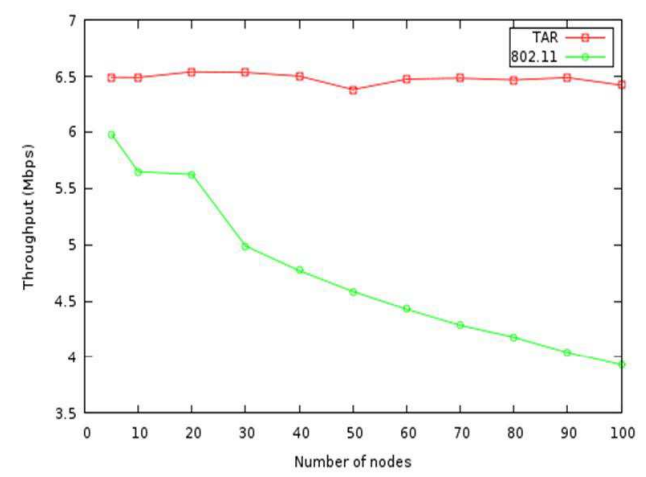

Fig. 4. Throughput comparison between IEEE 802.11 and TAR

throughput of TAR is about 5\% higher than that of IEEE 802.11 DCF mode. However the difference increases significantly with the number of nodes. This is due to TAR's coordinated channel access that succeeds in preserving the channel efficiency in high density situations. This is not the case of IEEE 802.11 where the throughput decreases significantly with the density increase. This is because of the high number of competing nodes that increase the collision rate and the bandwidth waste. TAR throughput does not dependent on the network density. This can be considered as a good advantage for vehicular communication in critical situation which require good bandwidth exploitation in high density scenarios.

\section{B. Collision rate}

Reducing the collisions in the network is one of the main objectives of TAR mechanism. The collision rate with TAR is negligible for a small number of nodes and considerably lower than that of IEEE 802.11 for dense networks. In fig. 5 we can see that the collision rate with TAR scheme for a high number of nodes is lower than 7\%. The high collision rate of IEEE 802.11 scheme is due to its random access scheme where collisions increase with the number of nodes whereas TAR's coordinated channel access succeeds in reducing the collision rate. Collisions can lead to a reception failure of a critical packet. With TAR mechanism collision rate is lower, that makes vehicular communication more efficient. This result makes TAR a good candidate for safety vehicular communications in critical situation.

\section{Fairness}

Short term fairness is an important evaluation criterion of a channel access mechanism as it provides the distribution of the channel time between the different contending nodes on a short timescale which is recommended in a vehicular communication. In vehicular 


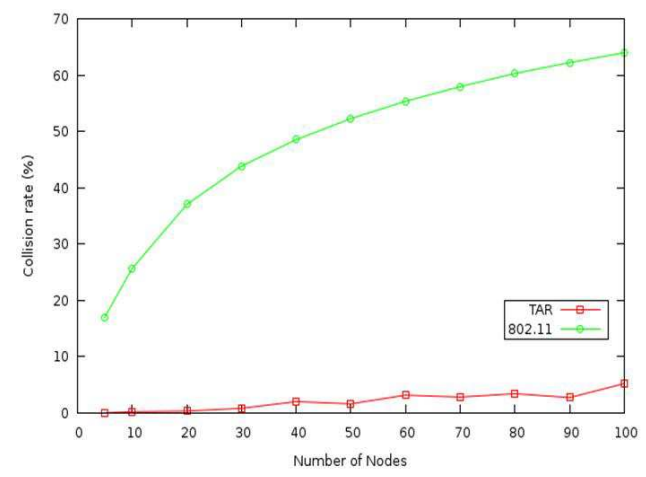

Fig. 5. Collision rates for IEEE 802.11 DCF mode and TAR

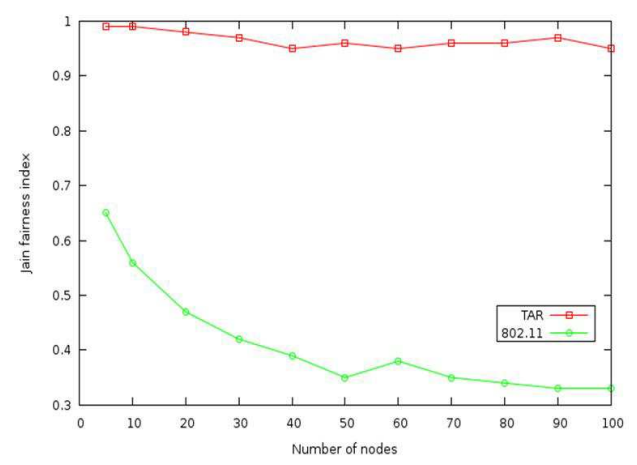

Fig. 6. Short term fairness comparison between TAR and IEEE 802.11

communications the number of contending vehicles that have an emergency packet can be very high. That's why in such situation a fair medium access with a short delay is required. Fig. 6 shows the Jain's fairness index of TAR and IEEE 802.11 in function of nodes number.

TAR succeeds in providing close to optimal short term fairness. This is due to the coordinated channel access cycle of TAR. When the number of nodes increases, the short term fairness index decreases slightly but remains close enough to the optimal. Contrarily to TAR, IEEE 802.11 suffers from short term unfairness that increases considerably with the number of nodes.

TAR provides fairness in access channel even for a high density network. This result is beneficial for critical situation in vehicular network. All vehicles could transmit their emergency packet in a high short term fairness.

\section{Inter transmission delay}

The inter-transmissions delay is the time difference observed between two consecutive transmissions of a given node. It indicates the time delay a node spends between two consecutive transmissions.

Table I presents the average values of the inter transmission delay of TAR and IEEE 802.11 for different network densities. The average inter-transmission delay is lower with TAR and the difference increases with the number of nodes. This is due to the coordination in the channel access that makes TAR more appropriate for time constrained applications like vehicular communication in critical situations. Vehicular network topology

TABLE I

AVERAGE VALUES OF THE INTER-TRANSMISSION DELAY IN MILLISECONDS

\begin{tabular}{|c|c|c|}
\hline NB nodes & TAR Avg. & 802.11 Avg. \\
\hline 40 & 73.030 & 99.673 \\
\hline 50 & 101.698 & 122.626 \\
\hline 60 & 110.038 & 161.008 \\
\hline 70 & 128.082 & 194.043 \\
\hline 80 & 146.855 & 228.431 \\
\hline 90 & 164.642 & 264.431 \\
\hline 100 & 184.970 & 302.313 \\
\hline
\end{tabular}

changes frequently. The time that communication link exist between two vehicles is brief. In addition, a vehicle involved in a critical situation might need to transmit its current status information repeatedly while it is engaged in the critical situation. This accentuates the need for an access scheme that provides high throughput with low inter transmission delays and high short term fairness which contribute to ensure efficient vehicular communications.

\section{CONCULSION}

In this paper we propose to apply TAR, a coordinated channel access mechanism, in a vehicular context. TAR scheme consists on allowing nodes to select and advertise a non selected Backoff value. By advertising the backoff selection, the network nodes avoid selecting already selected backoff values reducing thus the collision probability. We integrated TAR into NS-3 simulator and we evaluate its performances using scenario parameters appropriate to vehicular communication. Simulation results show that TAR performs better than IEEE 802.11 DCF. It improves throughput, reduces collisions and guarantees short term fairness in high network density. These results prove that TAR performs well in safety critical vehicular communication.

We are currently working on evaluating TAR using different vehicular contexts. Several scenarios of critical 
situations will be taken into account in order to study and evaluate its efficiency.

\section{REFERENCES}

[1] "IEEE P802.11p/D3.0, Draft Amendment for Wireless Access in Vehicular Environments (WAVE)," July 2007

[2] K. Bilstrup, E. Uhlemann, G. Strm and U. Bilstrup, "Evaluation of the IEEE 802.11p MAC method for Vehicle-to-Vehicle Communication," Vehicular Technology Conference, IEEE 68th, October 2008.

[3] B. Wehbi, A. Laouiti and A. Cavalli, "Transmit And Reserve (TAR): a Coordinated Channel Access for IEEE 802.11 Networks," In the proceedings of IEEE Wireless Communications and Networking Conference 2010 (WCNC 2010), Sydney, Australia, April 2010.

[4] Y. Wang, A. Ahmed, B. Krishnamachari and K. Psounis,"IEEE 802.11p Performance Evaluation and Protocol Enhancement," Proceedings of the 2008 IEEE International Conference on Vehicular Electronics and Safety Columbus, OH, USA, September 2008.

[5] P. Muhlethaler, A. Laouiti, Y. Toor, "Comparison of Flooding Techniques for Safety Application in VANETs," ITST '07. 7th International Conference on ITS In Telecommunications, August 2007.

[6] R. STANICA, E. Chaput and A.L. Beylot "Physical Carrier Sense in Vehicular Ad-Hoc Networks," Mobile Adhoc and Sensor Systems (MASS), 2011 IEEE 8th International Conference, October 2011

[7] J. Rezgui, S. Cherkaoui and O. Chakroun, "Deterministic Access for DSRC/802.11p Vehicular Safety Communication," Wireless Communications and Mobile Computing Conference (IWCMC), 7th International, August 2011.

[8] R. Jain, W. Hawe and D. Chiu, "A Quantitative Measure of Fairness and Discrimination for Resource Allocation in Shared Computer Systems," Technical report, Digital Equipment Corp., September 1984.

[9] “NS-3 tutorial Release ns-3.11,” Project ns-3, May 2011. 